\title{
El sueño en el crustáceo acocil
}

Fidel Ramón y Romero*

Universidad Nacional Autónoma de México, Facultad de Medicina, Departamento de Fisiología, Ciudad de México, México

\section{Resumen}

El sueño es definido como un estado de inconciencia, reducción en la actividad locomotora y despertar rápido, el cual está bien identificado en mamíferos, aves, reptiles y teleosteos. Comúnmente también es definido con registros eléctricos (electroencefalograma), los cuales solo están bien establecidos en mamíferos y, en cierta manera, en aves. Sin embargo, estados de sueño similares a los de mamíferos, excepto por los criterios eléctricos, parecen ocurrir en algunos invertebrados. Actualmente la mejor evidencia de sueño en invertebrados ha sido obtenida en el acocil. En los mamíferos, el sueño se caracteriza por un estado cerebral diferente al de la vigilia, que incluye un cambio a ondas lentas, lo que no se ha visto en Drosophila o abejas. Aquí mostramos que el acocil tiene un estado cerebral con umbral elevado a estímulos vibratorios, acompañado por una forma de actividad de ondas lentas en el cerebro, muy diferente al de la vigilia. Por lo tanto, el acocil puede experimentar un estado de sueño comparable al de los mamíferos.

PALABRAS CLAVE: Sueño. Actividad de ondas lentas en el cerebro. Crustáceo. Cerebro.

\begin{abstract}
Sleep is defined as a state of unconsciousness, reduced locomotive activity and rapid awakening, and is well established in mammals, birds, reptiles and teleosts. Commonly, it is also defined with electrical records (electroencephalogram), which are only well established in mammals and to some extent in birds. However, sleep states similar to those of mammals, except for electrical criteria, appear to occur in some invertebrates. Currently, the most compelling evidence of sleep in invertebrates has been obtained in the crayfish. In mammals, sleep is characterized by a brain state that is different from that of wakefulness, which includes a change to slow waves that has not been observed in Drosophila or bees. Herein, we show that the crayfish enters a brain state with a high threshold to vibratory stimuli, accompanied by a form of slow wave activity in the brain, quite different from that of wakefulness. Therefore, the crayfish can enter a state of sleep that is comparable to that of mammals.
\end{abstract}

KEY WORDS: Sleep. Slow wave activity in the brain. Crustacean. Brain.

\section{Introducción}

Desde hace mucho tiempo se conoce que los humanos y los animales con los que habitualmente estamos en contacto duermen. También se han realizado investigaciones en algunos animales invertebrados, si bien son de carácter conductual y no están dirigidas a la identificación de la razón del sueño.

Kayser y Steiner-Kaiser ${ }^{1,2}$ mostraron que, en ciertas condiciones, las abejas pierden el tono muscular, dejan caer las antenas y quedan quietas, conducta que interpretaron como estado de dormir. Por su parte, Tobler investigó numerosos animales invertebrados y
Gac Med Mex. 2019;155:537-540 


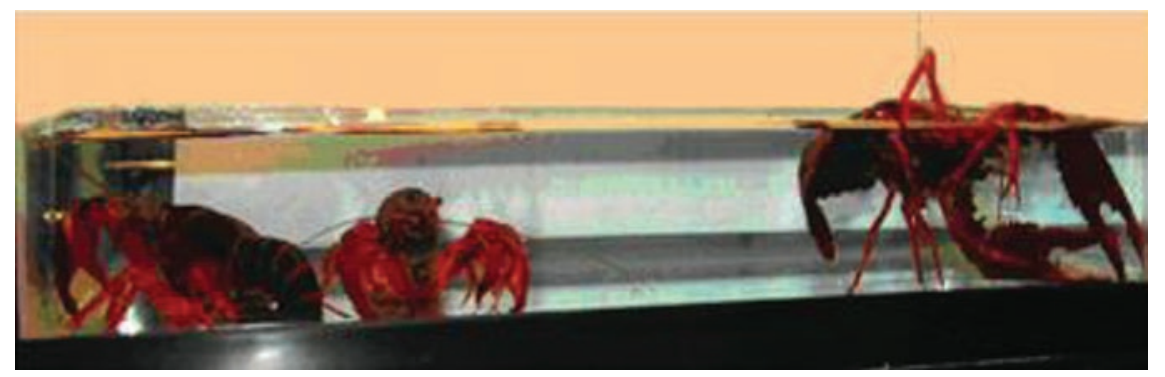

Figura 1. Acociles en un tanque con agua de la llave agitada vigorosamente y almacenada para su uso posterior. A la izquierda, dos acociles parados en el fondo del acuario. A la derecha, un acocil recostado en el agua deteniéndose con sus quelas.?

describió que pasaban varias horas del día inmóviles; explicó esa pasividad como la conducta de dormir. ${ }^{3-5}$ Todavía más, en numerosas investigaciones sobre la mosca Drosophila, los periodos de inmovilidad también fueron interpretados como equivalentes al sueño. Sin embargo, no existen evidencias de que esos animales tuvieran las características del sueño enunciadas claramente por Hobson. ${ }^{6}$

Nuestro grupo de trabajo no estaba particularmente interesado en el sueño en el acocil, sino en el procesamiento que hace el cerebro de las señales sensoriales y su efecto sobre la conducta. Para esa investigación se utilizó un crustáceo de agua dulce, el acocil, porque su tamaño facilita la implantación de electrodos en su cerebro y la realización de registros electrofisiológicos mientras el animal parece hacer una vida más o menos normal.

Los acociles eran mantenidos en acuarios con agua de la llave a la que se le quitó el cloro agitándola fuertemente ("agua vieja"), lo cual parecía no incomodar a los acociles, que pueden estar varios meses en estas condiciones, incluso si el agua se enturbia con sus excreciones; el agua era cambiada cuando sucedía esto último. En ocasiones, el agua en los acuarios se evaporaba y los animales tendían a ponerse de lado sobre la superficie cuando solo quedaban entre 5 y $7 \mathrm{~cm}$ de agua, conducta que observamos frecuentemente quienes trabajamos con acociles (Figura 1). Al no tener una explicación de esa posición, aventuramos varias explicaciones, entre ellas que quizá podrían estar durmiendo. Sin embargo, eso parecía tan improbable que abandonamos la idea.

En una ocasión, un acocil con electrodos implantados en el cerebro se colocó en esa posición de lado; en ese momento pensamos que sería oportuno registrar su actividad cerebral. Sorprendentemente, los registros en el osciloscopio mostraron ondas lentas con una frecuencia de aproximadamente $10 \mathrm{~Hz}$. Poco después, el animal recuperó su posición vertical en el
A

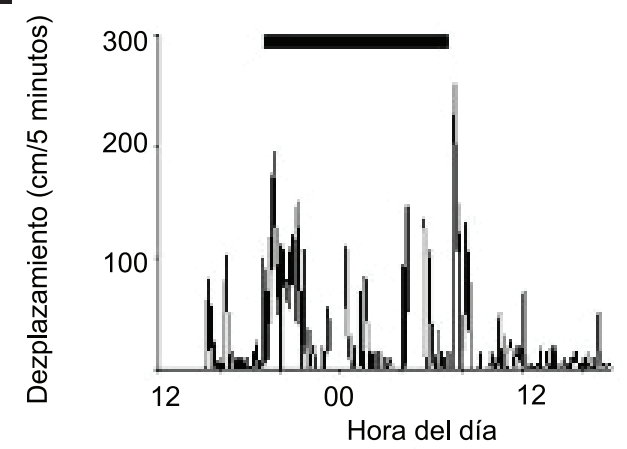

B

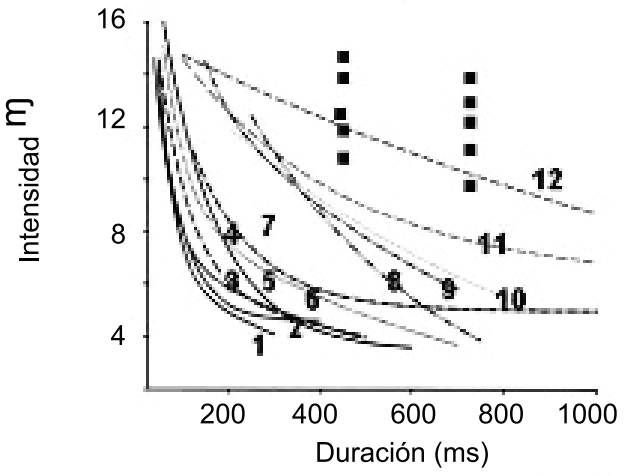

C

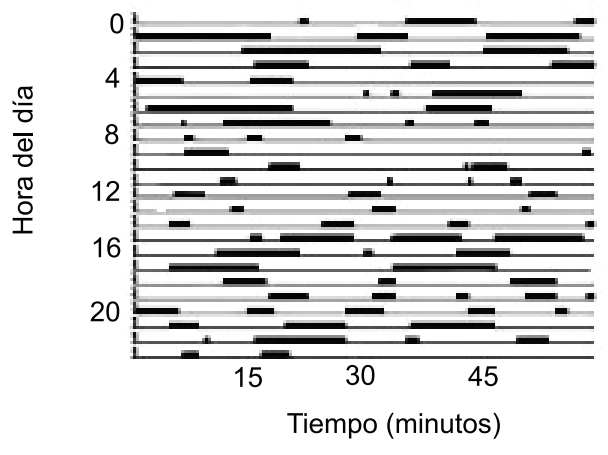

Figura 2. Registros de un acocil recostado sobre la superficie del agua. La barra negra indica el periodo de la noche. A: Tiempo durante el cual el acocil se desplaza alrededor del acuario. La mayor parte de los desplazamientos corresponde a las horas alrededor de las transiciones luz-oscuridad y oscuridad-luz; B: Curvas de intensidad-duración obtenidas al aplicar estímulos vibratorios cuando el animal está desplazándose (curvas 1-7). Las curvas 1-10 corresponden a estímulos aplicados a animales parados quietos (8-11) y la curva 12, a un animal recostado sobre el agua; C: Registros del periodo en el que el acocil se encuentra recostado sobre el agua. A y $C$ muestran un ejemplo entre 12 casos registrados. ${ }^{7}$ 

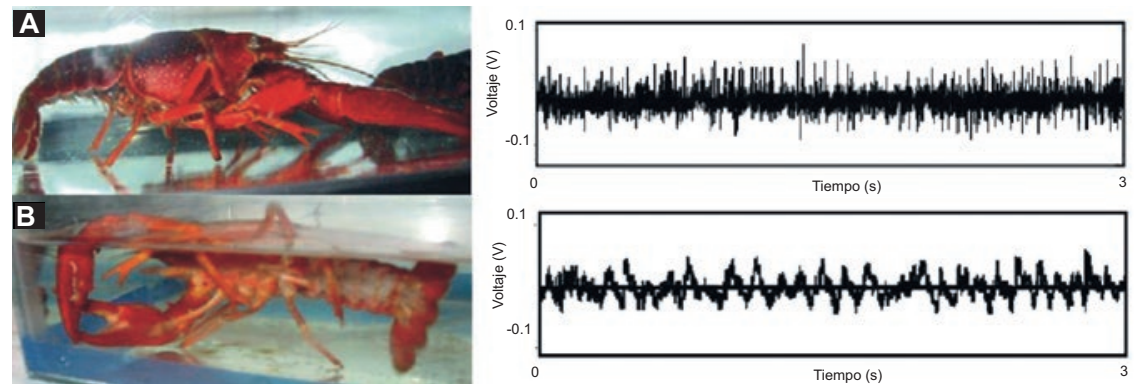

Figura 3. A: Fotografía y registro electrofisiológico de un acocil parado en el fondo de un acuario. Nótese las espigas de hasta $100 \mu \mathrm{V}$ de amplitud, correspondientes al registro de campo del cerebro; B: Acocil recostado de lado en la superficie del agua en un acuario y el registro de las ondas lentas en el cerebro cuando el animal se encuentra en esa posición.?
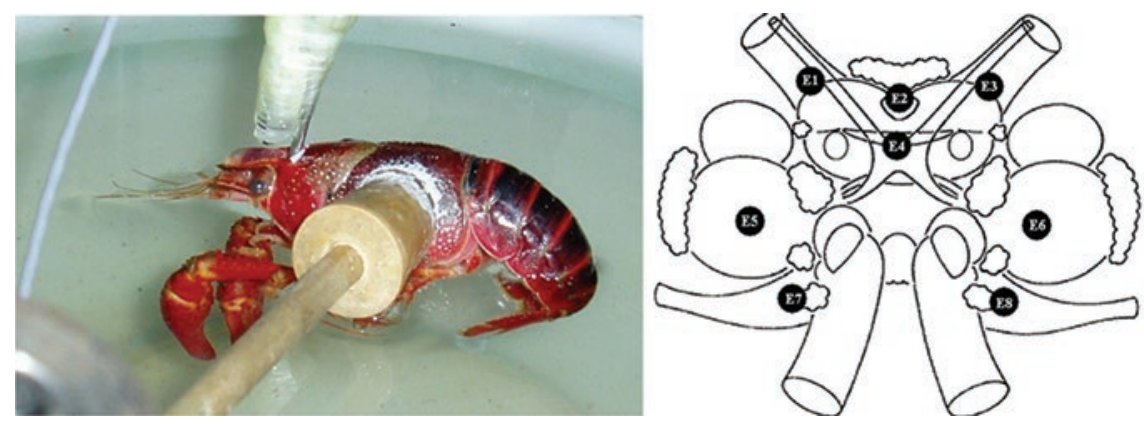

Figura 4. Acocil suspendido en el agua en una posición que permitía el registro electrofisiológico del cerebro, mientras el animal estaba libre para mover los apéndices. En el diagrama del cerebro se indican (con puntos oscuros simétricos del 1 al 8) las regiones registradas en el cerebro: E1 y E3, protocerebro lateral; E2, puente protocerebral; E4, complejo central del cerebro; E5 y E6, lóbulo accesorio del deutocerebro; E7 y E8, tritocerebro (imagen tomada de Mendoza-Ángeles et al.). ${ }^{8}$ La nomenclatura es de Sandeman et al. ${ }^{9}$

fondo del acuario y observamos la multitud de espigas características del registro normal del cerebro. Ante este hallazgo, decidimos esperar a que el acocil volviera a ponerse de lado para obtener nuevo registros. Hicimos las pruebas correspondientes a un animal durmiendo, para ver si el patrón correspondía a ese estado cerebral (Figura 2).

\section{Análisis de las señales}

Al principio, los registros fueron analizados mediante la transformada rápida de Fourier para obtener frecuencias, estrategia que resultó no ser la mejor para estudiar los registros encefalográficos, por lo que se optó por la transformada ondícula (wavelet transform).

Hay que recordar que la transformada de Fourier permite hacer el análisis de una serie de señales periódicas, pero solamente entrega un espacio de frecuencia e intensidad. En cambio, la transformada ondícula brinda un espacio mucho más completo, en el que coinciden la intensidad, la frecuencia y el tiempo, por lo que es más útil para identificar dónde y cuándo ocurren los cambios en frecuencia en los registros del cerebro.

\section{Registros de sueño}

Las primeras indicaciones de un cambio en el estado del cerebro del acocil fueron el cambio postural y la inmovilidad, así como el cambio en el tipo de señales, que pasaban de una cadena continúa de espigas entre 20 y $50 \mu \mathrm{V}$ de amplitud a una serie de ondas lentas entre 10 a $20 \mathrm{cps}$ (Figura 3).

En una siguiente etapa se mapeó el cerebro del acocil para localizar la región que produce las ondas lentas. El acocil fue colocado en un tanque con agua en el que fue fijado a un vástago dejando libres todos sus apéndices. Hicimos un agujero en el cefalotórax para exponer la superficie dorsal y anterior del cerebro, para escoger la región por registrar (Figura 4).

Para comparar los registros de sueño del acocil con los de otros animales, entre ellos los seres humanos, es necesario tener en cuenta diversos factores. Los seres humanos tienen dos tipos de sueño, uno llamado REM (rapid eye movements) y otro no-REM (sin movimientos oculares rápidos) durante el cual se producen las ensoñaciones. Aunque es posible que el acocil mueva alguno de sus apéndices, no es posible determinar si lo hace, por lo que este modelo sería aplicable solamente al estado de sueño no-REM. 


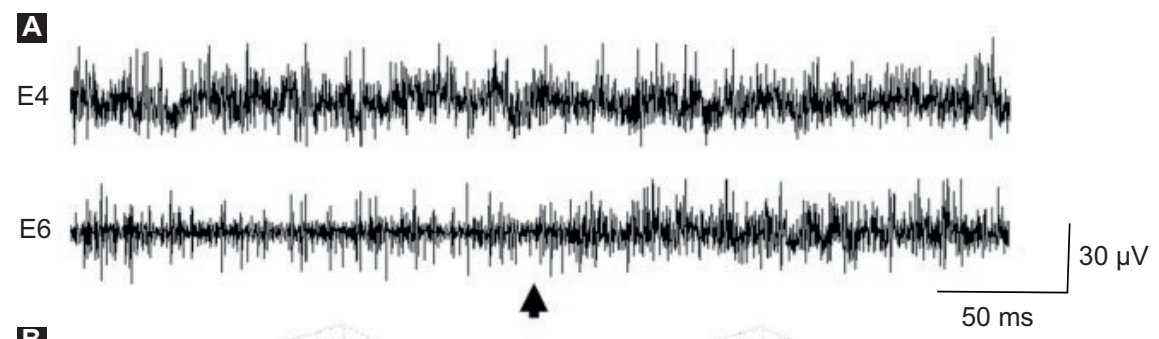

B
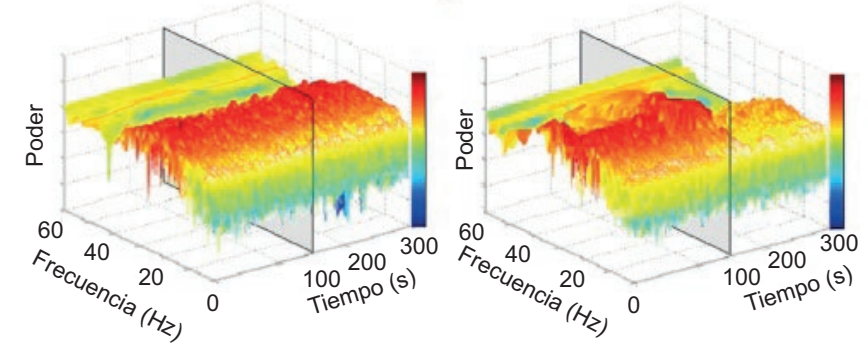

Figura 5. Transición del estado activo al sueño en un acocil suspendido. A: Actividad eléctrica registrada en el complejo central del cerebro de un acocil (E4) y en el deutocerebro (E6) durante la transición de vigilia al sueño; B: Análisis por transformada ondícula de los registros en E4 y E6 (los cuadros grises indican el momento de la transición). Antes de la transición (marcada por la flecha) no hay cambios en E4 (registro y análisis por transformada ondícula); en cambio, hay disminución clara en potencia en E6 (cuadro gris), correspondiendo con la aparición de las ondas lentas y el inicio del sueño (tomada de Mendoza-Ángeles et al. ${ }^{10}$ ).

En resumen, como es claro en la Figura 5, el origen de la transición de un acocil activo a uno dormido ocurre con el cambio de un registro dominado por espigas a uno con ondas lentas, lo que sucede en el complejo central del cerebro. Mientras tanto, otras regiones cerebrales continúan con registros dominados por espigas unitarias. El complejo central del acocil parece tener una posible homología con el de los insectos.

\section{Agradecimientos}

A la ingeniera Karina Mendoza, por su colaboración con los programas para analizar los registros por medio de transformada ondícula.

\section{Bibliografía}

1. Kaiser W, Steiner-Kaiser J. Neuronal correlates of sleep, wakefulness and arousal in a diurnal insect. Nature. 1983;301:707-709.

2. Kaiser W. Busy bees need rest, too. Behavioral and electromyographical sleep signs in honeybees. J Comp Physiol A. 1988:163:565-584.

3. Tobler I. Effect of forced locomotion on the rest-activity cycle of the cockroach. Behav Brain Res. 1983;8:351-360.

4. Tobler I, Stalder J. Rest in the scorpion. A sleep-like state? J Comp Physiol A. 1988;163:227-235.

5. Tobler I, Neuner-Jehle M. 24-h variation of vigilance in the cockroach Blaberus giganteus. J Sleep Res. 1992;1:231-239.

6. Hobson JA. Sleep is of the brain, by the brain and for the brain. Nature. 2005;437:1254-1256.

7. Ramón F, Hernández-Falcón J, Nguyen B, Bullock TH. Slow wave sleep in crayfish. Proc Natl Acad Sci U S A. 2004;101:11857-11861.

8. Mendoza-Ángeles K, Hernández-Falcón J, Cabrera A, Ramón F. Crayfish brain states characterization with wavelet transform. $2^{\text {nd }}$ International Conference on Electrical and Electronics Engineering and XI Conference on Electrical Engineering, Mexico City, Mexico, September 7-9, 2005.

9. Sandeman D, Sandeman R, Derby C, Schmidt M. Morphology of the brain of crayfish, crabs, and spiny lobsters: a common nomenclature for homologous structures. Biol Bull. 1992;183:304-326.

10. Mendoza-Ángeles K, Hernández-Falcón J, Ramón F. Slow waves during sleep in crayfish. Origin and spread. J Exp Biol. 2010;213:2154-2164. 